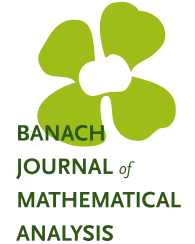

Banach J. Math. Anal. 10 (2016), no. 4, 671-685

http://dx.doi.org/10.1215/17358787-3649260

ISSN: $1735-8787$ (electronic)

http://projecteuclid.org/bjma

\title{
AN EXTENSION OF A THEOREM OF SCHOENBERG TO PRODUCTS OF SPHERES
}

\author{
J. C. GUELlA, V. A. MENEGATTO, and A. P. PERON* \\ Communicated by D. Han
}

\begin{abstract}
We present a characterization for the continuous, isotropic, and positive definite kernels on a product of spheres along the lines of a classical result of Schoenberg on positive definiteness on a single sphere. We also discuss a few issues regarding the characterization, including topics for future investigation.
\end{abstract}

\section{INTRODUCTION}

We consider the problem of characterizing positive definite kernels on a product of spheres. Our focus will be on continuous and isotropic kernels, keeping the setting originally adopted by Schoenberg in his influential work published in 1942 (see [20]).

As usual, let $S^{m}$ denote the unit sphere in the $(m+1)$-dimensional space $\mathbb{R}^{m+1}$, and let $S^{\infty}$ denote the unit sphere in the usual real $\ell^{2}$ space (here denoted by $\mathbb{R}^{\infty}$ for convenience). Throughout the present article, we will be dealing with real, continuous, and isotropic kernels on the product $S^{m} \times S^{M}, m, M=1,2, \ldots, \infty$. When speaking of continuity, we will assume that each sphere is endowed with its usual geodesic distance. The isotropy (zonality) of a kernel $K$ on $S^{m} \times S^{M}$ refers to the fact that

$$
K((x, z),(y, w))=f(x \cdot y, z \cdot w), \quad x, y \in S^{m}, z, w \in S^{M},
$$

Copyright 2016 by the Tusi Mathematical Research Group.

Received Jul. 1, 2015; Accepted Dec. 19, 2015.

${ }^{*}$ Corresponding author.

2010 Mathematics Subject Classification. Primary 43A35; Secondary 33C50, 33C55, 42A10, $42 \mathrm{~A} 82$.

Keywords. positive definiteness, spherical harmonics, isotropy, Gegenbauer polynomials, addition formula. 


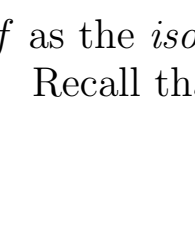

Banach J. Math. Anal. 10 (2016), no. 4, 671-685

http://dx.doi.org/10.1215/17358787-3649260

ISSN: $1735-8787$ (electronic)

http://projecteuclid.org/bjma

\title{
AN EXTENSION OF A THEOREM OF SCHOENBERG TO PRODUCTS OF SPHERES
}

\author{
J. C. GUELlA, V. A. MENEGATTO, and A. P. PERON* \\ Communicated by D. Han
}

\begin{abstract}
We present a characterization for the continuous, isotropic, and positive definite kernels on a product of spheres along the lines of a classical result of Schoenberg on positive definiteness on a single sphere. We also discuss a few issues regarding the characterization, including topics for future investigation.
\end{abstract}

\section{INTRODUCTION}

We consider the problem of characterizing positive definite kernels on a product of spheres. Our focus will be on continuous and isotropic kernels, keeping the setting originally adopted by Schoenberg in his influential work published in 1942 (see [20]).

As usual, let $S^{m}$ denote the unit sphere in the $(m+1)$-dimensional space $\mathbb{R}^{m+1}$, and let $S^{\infty}$ denote the unit sphere in the usual real $\ell^{2}$ space (here denoted by $\mathbb{R}^{\infty}$ for convenience). Throughout the present article, we will be dealing with real, continuous, and isotropic kernels on the product $S^{m} \times S^{M}, m, M=1,2, \ldots, \infty$. When speaking of continuity, we will assume that each sphere is endowed with its usual geodesic distance. The isotropy (zonality) of a kernel $K$ on $S^{m} \times S^{M}$ refers to the fact that

$$
K((x, z),(y, w))=f(x \cdot y, z \cdot w), \quad x, y \in S^{m}, z, w \in S^{M},
$$

Copyright 2016 by the Tusi Mathematical Research Group.

Received Jul. 1, 2015; Accepted Dec. 19, 2015.

${ }^{*}$ Corresponding author.

2010 Mathematics Subject Classification. Primary 43A35; Secondary 33C50, 33C55, 42A10, $42 \mathrm{~A} 82$.

Keywords. positive definiteness, spherical harmonics, isotropy, Gegenbauer polynomials, addition formula. 


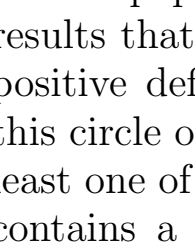

Banach J. Math. Anal. 10 (2016), no. 4, 671-685

http://dx.doi.org/10.1215/17358787-3649260

ISSN: $1735-8787$ (electronic)

http://projecteuclid.org/bjma

\title{
AN EXTENSION OF A THEOREM OF SCHOENBERG TO PRODUCTS OF SPHERES
}

\author{
J. C. GUELlA, V. A. MENEGATTO, and A. P. PERON* \\ Communicated by D. Han
}

\begin{abstract}
We present a characterization for the continuous, isotropic, and positive definite kernels on a product of spheres along the lines of a classical result of Schoenberg on positive definiteness on a single sphere. We also discuss a few issues regarding the characterization, including topics for future investigation.
\end{abstract}

\section{INTRODUCTION}

We consider the problem of characterizing positive definite kernels on a product of spheres. Our focus will be on continuous and isotropic kernels, keeping the setting originally adopted by Schoenberg in his influential work published in 1942 (see [20]).

As usual, let $S^{m}$ denote the unit sphere in the $(m+1)$-dimensional space $\mathbb{R}^{m+1}$, and let $S^{\infty}$ denote the unit sphere in the usual real $\ell^{2}$ space (here denoted by $\mathbb{R}^{\infty}$ for convenience). Throughout the present article, we will be dealing with real, continuous, and isotropic kernels on the product $S^{m} \times S^{M}, m, M=1,2, \ldots, \infty$. When speaking of continuity, we will assume that each sphere is endowed with its usual geodesic distance. The isotropy (zonality) of a kernel $K$ on $S^{m} \times S^{M}$ refers to the fact that

$$
K((x, z),(y, w))=f(x \cdot y, z \cdot w), \quad x, y \in S^{m}, z, w \in S^{M},
$$

Copyright 2016 by the Tusi Mathematical Research Group.

Received Jul. 1, 2015; Accepted Dec. 19, 2015.

${ }^{*}$ Corresponding author.

2010 Mathematics Subject Classification. Primary 43A35; Secondary 33C50, 33C55, 42A10, $42 \mathrm{~A} 82$.

Keywords. positive definiteness, spherical harmonics, isotropy, Gegenbauer polynomials, addition formula. 


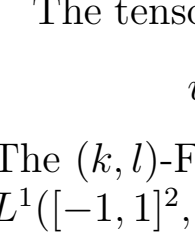

Banach J. Math. Anal. 10 (2016), no. 4, 671-685

http://dx.doi.org/10.1215/17358787-3649260

ISSN: $1735-8787$ (electronic)

http://projecteuclid.org/bjma

\title{
AN EXTENSION OF A THEOREM OF SCHOENBERG TO PRODUCTS OF SPHERES
}

\author{
J. C. GUELlA, V. A. MENEGATTO, and A. P. PERON* \\ Communicated by D. Han
}

\begin{abstract}
We present a characterization for the continuous, isotropic, and positive definite kernels on a product of spheres along the lines of a classical result of Schoenberg on positive definiteness on a single sphere. We also discuss a few issues regarding the characterization, including topics for future investigation.
\end{abstract}

\section{INTRODUCTION}

We consider the problem of characterizing positive definite kernels on a product of spheres. Our focus will be on continuous and isotropic kernels, keeping the setting originally adopted by Schoenberg in his influential work published in 1942 (see [20]).

As usual, let $S^{m}$ denote the unit sphere in the $(m+1)$-dimensional space $\mathbb{R}^{m+1}$, and let $S^{\infty}$ denote the unit sphere in the usual real $\ell^{2}$ space (here denoted by $\mathbb{R}^{\infty}$ for convenience). Throughout the present article, we will be dealing with real, continuous, and isotropic kernels on the product $S^{m} \times S^{M}, m, M=1,2, \ldots, \infty$. When speaking of continuity, we will assume that each sphere is endowed with its usual geodesic distance. The isotropy (zonality) of a kernel $K$ on $S^{m} \times S^{M}$ refers to the fact that

$$
K((x, z),(y, w))=f(x \cdot y, z \cdot w), \quad x, y \in S^{m}, z, w \in S^{M},
$$

Copyright 2016 by the Tusi Mathematical Research Group.

Received Jul. 1, 2015; Accepted Dec. 19, 2015.

${ }^{*}$ Corresponding author.

2010 Mathematics Subject Classification. Primary 43A35; Secondary 33C50, 33C55, 42A10, $42 \mathrm{~A} 82$.

Keywords. positive definiteness, spherical harmonics, isotropy, Gegenbauer polynomials, addition formula. 


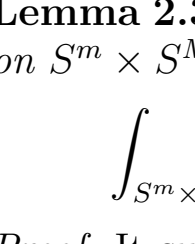

Banach J. Math. Anal. 10 (2016), no. 4, 671-685

http://dx.doi.org/10.1215/17358787-3649260

ISSN: $1735-8787$ (electronic)

http://projecteuclid.org/bjma

\title{
AN EXTENSION OF A THEOREM OF SCHOENBERG TO PRODUCTS OF SPHERES
}

\author{
J. C. GUELlA, V. A. MENEGATTO, and A. P. PERON* \\ Communicated by D. Han
}

\begin{abstract}
We present a characterization for the continuous, isotropic, and positive definite kernels on a product of spheres along the lines of a classical result of Schoenberg on positive definiteness on a single sphere. We also discuss a few issues regarding the characterization, including topics for future investigation.
\end{abstract}

\section{INTRODUCTION}

We consider the problem of characterizing positive definite kernels on a product of spheres. Our focus will be on continuous and isotropic kernels, keeping the setting originally adopted by Schoenberg in his influential work published in 1942 (see [20]).

As usual, let $S^{m}$ denote the unit sphere in the $(m+1)$-dimensional space $\mathbb{R}^{m+1}$, and let $S^{\infty}$ denote the unit sphere in the usual real $\ell^{2}$ space (here denoted by $\mathbb{R}^{\infty}$ for convenience). Throughout the present article, we will be dealing with real, continuous, and isotropic kernels on the product $S^{m} \times S^{M}, m, M=1,2, \ldots, \infty$. When speaking of continuity, we will assume that each sphere is endowed with its usual geodesic distance. The isotropy (zonality) of a kernel $K$ on $S^{m} \times S^{M}$ refers to the fact that

$$
K((x, z),(y, w))=f(x \cdot y, z \cdot w), \quad x, y \in S^{m}, z, w \in S^{M},
$$

Copyright 2016 by the Tusi Mathematical Research Group.

Received Jul. 1, 2015; Accepted Dec. 19, 2015.

${ }^{*}$ Corresponding author.

2010 Mathematics Subject Classification. Primary 43A35; Secondary 33C50, 33C55, 42A10, $42 \mathrm{~A} 82$.

Keywords. positive definiteness, spherical harmonics, isotropy, Gegenbauer polynomials, addition formula. 


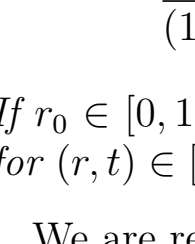

Banach J. Math. Anal. 10 (2016), no. 4, 671-685

http://dx.doi.org/10.1215/17358787-3649260

ISSN: $1735-8787$ (electronic)

http://projecteuclid.org/bjma

\title{
AN EXTENSION OF A THEOREM OF SCHOENBERG TO PRODUCTS OF SPHERES
}

\author{
J. C. GUELlA, V. A. MENEGATTO, and A. P. PERON* \\ Communicated by D. Han
}

\begin{abstract}
We present a characterization for the continuous, isotropic, and positive definite kernels on a product of spheres along the lines of a classical result of Schoenberg on positive definiteness on a single sphere. We also discuss a few issues regarding the characterization, including topics for future investigation.
\end{abstract}

\section{INTRODUCTION}

We consider the problem of characterizing positive definite kernels on a product of spheres. Our focus will be on continuous and isotropic kernels, keeping the setting originally adopted by Schoenberg in his influential work published in 1942 (see [20]).

As usual, let $S^{m}$ denote the unit sphere in the $(m+1)$-dimensional space $\mathbb{R}^{m+1}$, and let $S^{\infty}$ denote the unit sphere in the usual real $\ell^{2}$ space (here denoted by $\mathbb{R}^{\infty}$ for convenience). Throughout the present article, we will be dealing with real, continuous, and isotropic kernels on the product $S^{m} \times S^{M}, m, M=1,2, \ldots, \infty$. When speaking of continuity, we will assume that each sphere is endowed with its usual geodesic distance. The isotropy (zonality) of a kernel $K$ on $S^{m} \times S^{M}$ refers to the fact that

$$
K((x, z),(y, w))=f(x \cdot y, z \cdot w), \quad x, y \in S^{m}, z, w \in S^{M},
$$

Copyright 2016 by the Tusi Mathematical Research Group.

Received Jul. 1, 2015; Accepted Dec. 19, 2015.

${ }^{*}$ Corresponding author.

2010 Mathematics Subject Classification. Primary 43A35; Secondary 33C50, 33C55, 42A10, $42 \mathrm{~A} 82$.

Keywords. positive definiteness, spherical harmonics, isotropy, Gegenbauer polynomials, addition formula. 


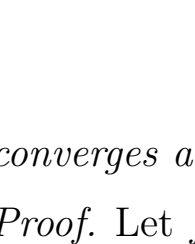

Banach J. Math. Anal. 10 (2016), no. 4, 671-685

http://dx.doi.org/10.1215/17358787-3649260

ISSN: $1735-8787$ (electronic)

http://projecteuclid.org/bjma

\title{
AN EXTENSION OF A THEOREM OF SCHOENBERG TO PRODUCTS OF SPHERES
}

\author{
J. C. GUELlA, V. A. MENEGATTO, and A. P. PERON* \\ Communicated by D. Han
}

\begin{abstract}
We present a characterization for the continuous, isotropic, and positive definite kernels on a product of spheres along the lines of a classical result of Schoenberg on positive definiteness on a single sphere. We also discuss a few issues regarding the characterization, including topics for future investigation.
\end{abstract}

\section{INTRODUCTION}

We consider the problem of characterizing positive definite kernels on a product of spheres. Our focus will be on continuous and isotropic kernels, keeping the setting originally adopted by Schoenberg in his influential work published in 1942 (see [20]).

As usual, let $S^{m}$ denote the unit sphere in the $(m+1)$-dimensional space $\mathbb{R}^{m+1}$, and let $S^{\infty}$ denote the unit sphere in the usual real $\ell^{2}$ space (here denoted by $\mathbb{R}^{\infty}$ for convenience). Throughout the present article, we will be dealing with real, continuous, and isotropic kernels on the product $S^{m} \times S^{M}, m, M=1,2, \ldots, \infty$. When speaking of continuity, we will assume that each sphere is endowed with its usual geodesic distance. The isotropy (zonality) of a kernel $K$ on $S^{m} \times S^{M}$ refers to the fact that

$$
K((x, z),(y, w))=f(x \cdot y, z \cdot w), \quad x, y \in S^{m}, z, w \in S^{M},
$$

Copyright 2016 by the Tusi Mathematical Research Group.

Received Jul. 1, 2015; Accepted Dec. 19, 2015.

${ }^{*}$ Corresponding author.

2010 Mathematics Subject Classification. Primary 43A35; Secondary 33C50, 33C55, 42A10, $42 \mathrm{~A} 82$.

Keywords. positive definiteness, spherical harmonics, isotropy, Gegenbauer polynomials, addition formula. 


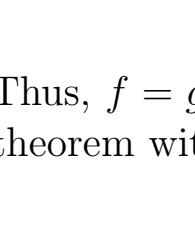

Banach J. Math. Anal. 10 (2016), no. 4, 671-685

http://dx.doi.org/10.1215/17358787-3649260

ISSN: $1735-8787$ (electronic)

http://projecteuclid.org/bjma

\title{
AN EXTENSION OF A THEOREM OF SCHOENBERG TO PRODUCTS OF SPHERES
}

\author{
J. C. GUELlA, V. A. MENEGATTO, and A. P. PERON* \\ Communicated by D. Han
}

\begin{abstract}
We present a characterization for the continuous, isotropic, and positive definite kernels on a product of spheres along the lines of a classical result of Schoenberg on positive definiteness on a single sphere. We also discuss a few issues regarding the characterization, including topics for future investigation.
\end{abstract}

\section{INTRODUCTION}

We consider the problem of characterizing positive definite kernels on a product of spheres. Our focus will be on continuous and isotropic kernels, keeping the setting originally adopted by Schoenberg in his influential work published in 1942 (see [20]).

As usual, let $S^{m}$ denote the unit sphere in the $(m+1)$-dimensional space $\mathbb{R}^{m+1}$, and let $S^{\infty}$ denote the unit sphere in the usual real $\ell^{2}$ space (here denoted by $\mathbb{R}^{\infty}$ for convenience). Throughout the present article, we will be dealing with real, continuous, and isotropic kernels on the product $S^{m} \times S^{M}, m, M=1,2, \ldots, \infty$. When speaking of continuity, we will assume that each sphere is endowed with its usual geodesic distance. The isotropy (zonality) of a kernel $K$ on $S^{m} \times S^{M}$ refers to the fact that

$$
K((x, z),(y, w))=f(x \cdot y, z \cdot w), \quad x, y \in S^{m}, z, w \in S^{M},
$$

Copyright 2016 by the Tusi Mathematical Research Group.

Received Jul. 1, 2015; Accepted Dec. 19, 2015.

${ }^{*}$ Corresponding author.

2010 Mathematics Subject Classification. Primary 43A35; Secondary 33C50, 33C55, 42A10, $42 \mathrm{~A} 82$.

Keywords. positive definiteness, spherical harmonics, isotropy, Gegenbauer polynomials, addition formula. 


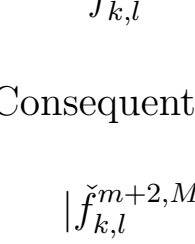

Banach J. Math. Anal. 10 (2016), no. 4, 671-685

http://dx.doi.org/10.1215/17358787-3649260

ISSN: $1735-8787$ (electronic)

http://projecteuclid.org/bjma

\title{
AN EXTENSION OF A THEOREM OF SCHOENBERG TO PRODUCTS OF SPHERES
}

\author{
J. C. GUELlA, V. A. MENEGATTO, and A. P. PERON* \\ Communicated by D. Han
}

\begin{abstract}
We present a characterization for the continuous, isotropic, and positive definite kernels on a product of spheres along the lines of a classical result of Schoenberg on positive definiteness on a single sphere. We also discuss a few issues regarding the characterization, including topics for future investigation.
\end{abstract}

\section{INTRODUCTION}

We consider the problem of characterizing positive definite kernels on a product of spheres. Our focus will be on continuous and isotropic kernels, keeping the setting originally adopted by Schoenberg in his influential work published in 1942 (see [20]).

As usual, let $S^{m}$ denote the unit sphere in the $(m+1)$-dimensional space $\mathbb{R}^{m+1}$, and let $S^{\infty}$ denote the unit sphere in the usual real $\ell^{2}$ space (here denoted by $\mathbb{R}^{\infty}$ for convenience). Throughout the present article, we will be dealing with real, continuous, and isotropic kernels on the product $S^{m} \times S^{M}, m, M=1,2, \ldots, \infty$. When speaking of continuity, we will assume that each sphere is endowed with its usual geodesic distance. The isotropy (zonality) of a kernel $K$ on $S^{m} \times S^{M}$ refers to the fact that

$$
K((x, z),(y, w))=f(x \cdot y, z \cdot w), \quad x, y \in S^{m}, z, w \in S^{M},
$$

Copyright 2016 by the Tusi Mathematical Research Group.

Received Jul. 1, 2015; Accepted Dec. 19, 2015.

${ }^{*}$ Corresponding author.

2010 Mathematics Subject Classification. Primary 43A35; Secondary 33C50, 33C55, 42A10, $42 \mathrm{~A} 82$.

Keywords. positive definiteness, spherical harmonics, isotropy, Gegenbauer polynomials, addition formula. 


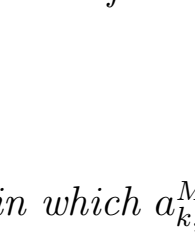

Banach J. Math. Anal. 10 (2016), no. 4, 671-685

http://dx.doi.org/10.1215/17358787-3649260

ISSN: $1735-8787$ (electronic)

http://projecteuclid.org/bjma

\title{
AN EXTENSION OF A THEOREM OF SCHOENBERG TO PRODUCTS OF SPHERES
}

\author{
J. C. GUELlA, V. A. MENEGATTO, and A. P. PERON* \\ Communicated by D. Han
}

\begin{abstract}
We present a characterization for the continuous, isotropic, and positive definite kernels on a product of spheres along the lines of a classical result of Schoenberg on positive definiteness on a single sphere. We also discuss a few issues regarding the characterization, including topics for future investigation.
\end{abstract}

\section{INTRODUCTION}

We consider the problem of characterizing positive definite kernels on a product of spheres. Our focus will be on continuous and isotropic kernels, keeping the setting originally adopted by Schoenberg in his influential work published in 1942 (see [20]).

As usual, let $S^{m}$ denote the unit sphere in the $(m+1)$-dimensional space $\mathbb{R}^{m+1}$, and let $S^{\infty}$ denote the unit sphere in the usual real $\ell^{2}$ space (here denoted by $\mathbb{R}^{\infty}$ for convenience). Throughout the present article, we will be dealing with real, continuous, and isotropic kernels on the product $S^{m} \times S^{M}, m, M=1,2, \ldots, \infty$. When speaking of continuity, we will assume that each sphere is endowed with its usual geodesic distance. The isotropy (zonality) of a kernel $K$ on $S^{m} \times S^{M}$ refers to the fact that

$$
K((x, z),(y, w))=f(x \cdot y, z \cdot w), \quad x, y \in S^{m}, z, w \in S^{M},
$$

Copyright 2016 by the Tusi Mathematical Research Group.

Received Jul. 1, 2015; Accepted Dec. 19, 2015.

${ }^{*}$ Corresponding author.

2010 Mathematics Subject Classification. Primary 43A35; Secondary 33C50, 33C55, 42A10, $42 \mathrm{~A} 82$.

Keywords. positive definiteness, spherical harmonics, isotropy, Gegenbauer polynomials, addition formula. 


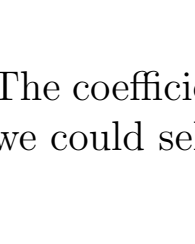

Banach J. Math. Anal. 10 (2016), no. 4, 671-685

http://dx.doi.org/10.1215/17358787-3649260

ISSN: $1735-8787$ (electronic)

http://projecteuclid.org/bjma

\title{
AN EXTENSION OF A THEOREM OF SCHOENBERG TO PRODUCTS OF SPHERES
}

\author{
J. C. GUELlA, V. A. MENEGATTO, and A. P. PERON* \\ Communicated by D. Han
}

\begin{abstract}
We present a characterization for the continuous, isotropic, and positive definite kernels on a product of spheres along the lines of a classical result of Schoenberg on positive definiteness on a single sphere. We also discuss a few issues regarding the characterization, including topics for future investigation.
\end{abstract}

\section{INTRODUCTION}

We consider the problem of characterizing positive definite kernels on a product of spheres. Our focus will be on continuous and isotropic kernels, keeping the setting originally adopted by Schoenberg in his influential work published in 1942 (see [20]).

As usual, let $S^{m}$ denote the unit sphere in the $(m+1)$-dimensional space $\mathbb{R}^{m+1}$, and let $S^{\infty}$ denote the unit sphere in the usual real $\ell^{2}$ space (here denoted by $\mathbb{R}^{\infty}$ for convenience). Throughout the present article, we will be dealing with real, continuous, and isotropic kernels on the product $S^{m} \times S^{M}, m, M=1,2, \ldots, \infty$. When speaking of continuity, we will assume that each sphere is endowed with its usual geodesic distance. The isotropy (zonality) of a kernel $K$ on $S^{m} \times S^{M}$ refers to the fact that

$$
K((x, z),(y, w))=f(x \cdot y, z \cdot w), \quad x, y \in S^{m}, z, w \in S^{M},
$$

Copyright 2016 by the Tusi Mathematical Research Group.

Received Jul. 1, 2015; Accepted Dec. 19, 2015.

${ }^{*}$ Corresponding author.

2010 Mathematics Subject Classification. Primary 43A35; Secondary 33C50, 33C55, 42A10, $42 \mathrm{~A} 82$.

Keywords. positive definiteness, spherical harmonics, isotropy, Gegenbauer polynomials, addition formula. 


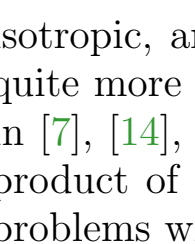

Banach J. Math. Anal. 10 (2016), no. 4, 671-685

http://dx.doi.org/10.1215/17358787-3649260

ISSN: $1735-8787$ (electronic)

http://projecteuclid.org/bjma

\title{
AN EXTENSION OF A THEOREM OF SCHOENBERG TO PRODUCTS OF SPHERES
}

\author{
J. C. GUELlA, V. A. MENEGATTO, and A. P. PERON* \\ Communicated by D. Han
}

\begin{abstract}
We present a characterization for the continuous, isotropic, and positive definite kernels on a product of spheres along the lines of a classical result of Schoenberg on positive definiteness on a single sphere. We also discuss a few issues regarding the characterization, including topics for future investigation.
\end{abstract}

\section{INTRODUCTION}

We consider the problem of characterizing positive definite kernels on a product of spheres. Our focus will be on continuous and isotropic kernels, keeping the setting originally adopted by Schoenberg in his influential work published in 1942 (see [20]).

As usual, let $S^{m}$ denote the unit sphere in the $(m+1)$-dimensional space $\mathbb{R}^{m+1}$, and let $S^{\infty}$ denote the unit sphere in the usual real $\ell^{2}$ space (here denoted by $\mathbb{R}^{\infty}$ for convenience). Throughout the present article, we will be dealing with real, continuous, and isotropic kernels on the product $S^{m} \times S^{M}, m, M=1,2, \ldots, \infty$. When speaking of continuity, we will assume that each sphere is endowed with its usual geodesic distance. The isotropy (zonality) of a kernel $K$ on $S^{m} \times S^{M}$ refers to the fact that

$$
K((x, z),(y, w))=f(x \cdot y, z \cdot w), \quad x, y \in S^{m}, z, w \in S^{M},
$$

Copyright 2016 by the Tusi Mathematical Research Group.

Received Jul. 1, 2015; Accepted Dec. 19, 2015.

${ }^{*}$ Corresponding author.

2010 Mathematics Subject Classification. Primary 43A35; Secondary 33C50, 33C55, 42A10, $42 \mathrm{~A} 82$.

Keywords. positive definiteness, spherical harmonics, isotropy, Gegenbauer polynomials, addition formula. 


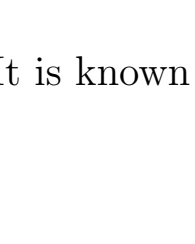

Banach J. Math. Anal. 10 (2016), no. 4, 671-685

http://dx.doi.org/10.1215/17358787-3649260

ISSN: $1735-8787$ (electronic)

http://projecteuclid.org/bjma

\title{
AN EXTENSION OF A THEOREM OF SCHOENBERG TO PRODUCTS OF SPHERES
}

\author{
J. C. GUELlA, V. A. MENEGATTO, and A. P. PERON* \\ Communicated by D. Han
}

\begin{abstract}
We present a characterization for the continuous, isotropic, and positive definite kernels on a product of spheres along the lines of a classical result of Schoenberg on positive definiteness on a single sphere. We also discuss a few issues regarding the characterization, including topics for future investigation.
\end{abstract}

\section{INTRODUCTION}

We consider the problem of characterizing positive definite kernels on a product of spheres. Our focus will be on continuous and isotropic kernels, keeping the setting originally adopted by Schoenberg in his influential work published in 1942 (see [20]).

As usual, let $S^{m}$ denote the unit sphere in the $(m+1)$-dimensional space $\mathbb{R}^{m+1}$, and let $S^{\infty}$ denote the unit sphere in the usual real $\ell^{2}$ space (here denoted by $\mathbb{R}^{\infty}$ for convenience). Throughout the present article, we will be dealing with real, continuous, and isotropic kernels on the product $S^{m} \times S^{M}, m, M=1,2, \ldots, \infty$. When speaking of continuity, we will assume that each sphere is endowed with its usual geodesic distance. The isotropy (zonality) of a kernel $K$ on $S^{m} \times S^{M}$ refers to the fact that

$$
K((x, z),(y, w))=f(x \cdot y, z \cdot w), \quad x, y \in S^{m}, z, w \in S^{M},
$$

Copyright 2016 by the Tusi Mathematical Research Group.

Received Jul. 1, 2015; Accepted Dec. 19, 2015.

${ }^{*}$ Corresponding author.

2010 Mathematics Subject Classification. Primary 43A35; Secondary 33C50, 33C55, 42A10, $42 \mathrm{~A} 82$.

Keywords. positive definiteness, spherical harmonics, isotropy, Gegenbauer polynomials, addition formula. 


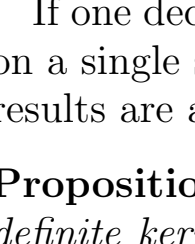

Banach J. Math. Anal. 10 (2016), no. 4, 671-685

http://dx.doi.org/10.1215/17358787-3649260

ISSN: $1735-8787$ (electronic)

http://projecteuclid.org/bjma

\title{
AN EXTENSION OF A THEOREM OF SCHOENBERG TO PRODUCTS OF SPHERES
}

\author{
J. C. GUELlA, V. A. MENEGATTO, and A. P. PERON* \\ Communicated by D. Han
}

\begin{abstract}
We present a characterization for the continuous, isotropic, and positive definite kernels on a product of spheres along the lines of a classical result of Schoenberg on positive definiteness on a single sphere. We also discuss a few issues regarding the characterization, including topics for future investigation.
\end{abstract}

\section{INTRODUCTION}

We consider the problem of characterizing positive definite kernels on a product of spheres. Our focus will be on continuous and isotropic kernels, keeping the setting originally adopted by Schoenberg in his influential work published in 1942 (see [20]).

As usual, let $S^{m}$ denote the unit sphere in the $(m+1)$-dimensional space $\mathbb{R}^{m+1}$, and let $S^{\infty}$ denote the unit sphere in the usual real $\ell^{2}$ space (here denoted by $\mathbb{R}^{\infty}$ for convenience). Throughout the present article, we will be dealing with real, continuous, and isotropic kernels on the product $S^{m} \times S^{M}, m, M=1,2, \ldots, \infty$. When speaking of continuity, we will assume that each sphere is endowed with its usual geodesic distance. The isotropy (zonality) of a kernel $K$ on $S^{m} \times S^{M}$ refers to the fact that

$$
K((x, z),(y, w))=f(x \cdot y, z \cdot w), \quad x, y \in S^{m}, z, w \in S^{M},
$$

Copyright 2016 by the Tusi Mathematical Research Group.

Received Jul. 1, 2015; Accepted Dec. 19, 2015.

${ }^{*}$ Corresponding author.

2010 Mathematics Subject Classification. Primary 43A35; Secondary 33C50, 33C55, 42A10, $42 \mathrm{~A} 82$.

Keywords. positive definiteness, spherical harmonics, isotropy, Gegenbauer polynomials, addition formula. 


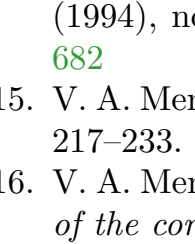

Banach J. Math. Anal. 10 (2016), no. 4, 671-685

http://dx.doi.org/10.1215/17358787-3649260

ISSN: $1735-8787$ (electronic)

http://projecteuclid.org/bjma

\title{
AN EXTENSION OF A THEOREM OF SCHOENBERG TO PRODUCTS OF SPHERES
}

\author{
J. C. GUELlA, V. A. MENEGATTO, and A. P. PERON* \\ Communicated by D. Han
}

\begin{abstract}
We present a characterization for the continuous, isotropic, and positive definite kernels on a product of spheres along the lines of a classical result of Schoenberg on positive definiteness on a single sphere. We also discuss a few issues regarding the characterization, including topics for future investigation.
\end{abstract}

\section{INTRODUCTION}

We consider the problem of characterizing positive definite kernels on a product of spheres. Our focus will be on continuous and isotropic kernels, keeping the setting originally adopted by Schoenberg in his influential work published in 1942 (see [20]).

As usual, let $S^{m}$ denote the unit sphere in the $(m+1)$-dimensional space $\mathbb{R}^{m+1}$, and let $S^{\infty}$ denote the unit sphere in the usual real $\ell^{2}$ space (here denoted by $\mathbb{R}^{\infty}$ for convenience). Throughout the present article, we will be dealing with real, continuous, and isotropic kernels on the product $S^{m} \times S^{M}, m, M=1,2, \ldots, \infty$. When speaking of continuity, we will assume that each sphere is endowed with its usual geodesic distance. The isotropy (zonality) of a kernel $K$ on $S^{m} \times S^{M}$ refers to the fact that

$$
K((x, z),(y, w))=f(x \cdot y, z \cdot w), \quad x, y \in S^{m}, z, w \in S^{M},
$$

Copyright 2016 by the Tusi Mathematical Research Group.

Received Jul. 1, 2015; Accepted Dec. 19, 2015.

${ }^{*}$ Corresponding author.

2010 Mathematics Subject Classification. Primary 43A35; Secondary 33C50, 33C55, 42A10, $42 \mathrm{~A} 82$.

Keywords. positive definiteness, spherical harmonics, isotropy, Gegenbauer polynomials, addition formula. 